\title{
Proposed New Framework Scheme for Path Loss in Wireless Body Area Network
}

\author{
Israa Al_Barazanchi ${ }^{1}$, Yitong Niu ${ }^{2}$, Haider Rasheed Abdulshaheed ${ }^{3}$, \\ Wahidah Hashim ${ }^{1}$, Ammar Ahmed Alkahtani ${ }^{4}$, Elika Daghighi ${ }^{5}$, \\ Zahraa A. Jaaz ${ }^{6,1}$, Shihab A. Shawkat ${ }^{7}$, Hafiz Tayyab Rauf ${ }^{8}$ \\ ${ }^{1}$ College of Computing and Informatics, Universiti Tenaga Nasional, Malaysia \\ ${ }^{2}$ Belarusian-Russian University, Mira Avenue 43, Mogilev, 212000, Republic of Belarus \\ ${ }^{3}$ Computer Engineering Techniques Department, Baghdad College of Economic Sciences University, Baghdad, Iraq \\ ${ }^{4}$ Institute of Sustainable Energy, Universiti Tenga Nasional, Kajang, 43000, Selangor, Malaysia \\ ${ }^{5}$ Technical and Vocational University, Tehran, Iran \\ ${ }^{6}$ College of Science, Computer Department, AlNahrain University, Baghdad \\ ${ }^{7}$ University of Samarra, Iraq \\ ${ }^{8}$ Centre for Smart Systems, AI and Cybersecurity, Staffordshire University, Stoke-on-Trent, United Kingdom
}

\author{
*Corresponding Author: Israa Al_Barazanchi
}

DOI: https://doi.org/10.52866/ijcsm.2022.01.01.002

Received October 2021; Accepted November 2021; Available online January 2022

\begin{abstract}
Recent technical developments in wi-fi networking, microelectronic integration and programming, sensors and the Internet have enabled us to create and enforce a range of new framework schemes to fulfil the necessities of healthcare-related wireless body area network (WBAN). WBAN sensors continually screen and measure patients' indispensable signs and symptoms, and relay them to scientific monitoring for diagnosis. WBAN has a range of applications, the most necessary of which is to help patients suffering diseases to stay alive. The quality instance is the coronary heart implant sensor, whose video display unit monitors coronary heart sign and continuously transmits it. This setup eliminates the need for patients to visit the medical doctor frequently. Instead, they can take a seat at home and acquire an analysis and prescription for the disease. Today, a sizable effort is being made to increase low-power sensors and gadgets for utility in WBAN. A new framework scheme that addresses route loss in WBAN and discusses its penalties in depth is endorsed in this paper. The new framework scheme is applied to three case scenarios to obtain parameters by measuring vital information about the human body. On-body and intrabody conversation simulations are conducted. On-body conversation findings show that the route loss between transmitter and receiver rises with growing distance and frequency.
\end{abstract}

Keywords: WBAN; MICS band; communication system; path loss; body area networks; CCU; monitoring health care environmen

\section{INTRODUCTION}

Wireless body area network (WBAN) functions in the scientific location consist of wearable and implanted sensor nodes that gather organic records from the human body. These statistics are wirelessly dispatched throughout brief distances to a managed device. The management system can also be worn on the physique or placed in a handy vicinity without problems [1]. The sensor electronics ought to be successful in detecting clinical pulses such as electrocardiograms, photoplethysmograms and electroencephalograms, as nicely as pulse rate, pressure and temperature. Additionally, it is successful in being shrivelled and has a low electricity consumption [2]. The manipulated device's facts are dispatched

*Corresponding author: israa44444@gmail.com , israa.albarazanchi@baghdadcollege.edu.iq 
to far-flung places for therapeutic and diagnostic functions using a wi-fi physique region network. This system is enabled by the incorporation of a wireless network meant for long-range transmissions [3]. The sensor node features and sensor schemes for information via negation used in WBAN and the loss problem are explained; the communications of sensor node in the human body is explained in detail, and the simulations for in-body and on-body communication are discussed. The path loss between transmitter and receiver rises with distance and frequency in on- body verbal exchange [4]. The WBAN channel mannequin is examined, alongside numerous modulation schemes that apply to the WBAN healthcare setting, as nicely as their OMNET++ simulations. The next chapter studies the published work for the same author of this paper [5] and provides an efficient solution for data loss by designing a new framework scheme under the name of identification key scheme. After determining essential routing protocols on the use of handy tools, a real-world case, in which various sufferers or a single affected person transmits to one or extra receiver units simultaneously, is carried out and evaluated. Wireless physique location networks have a range of applications, which include wearable and implanted sensor nodes that observe organic records about the human physique [2] This record is dispatched wirelessly to the manipulating system throughout quick distances. The main trouble with WBAN is fact loss or corruption throughout transmission; this area nonetheless requires a new algorithm or schemes to show the effectivity of receiving entire facts without loss; the loss is induced through free-space harm to the propagating signal, together with refraction, attenuation, absorption and reflection.

The goal of this paper is to extend the study [5] and investigate, evaluate and unravel the route loss problem in WBAN to meet the healthcare needs in a healthcare setting. This lookup establishes a clear direction ahead for determining the crucial scheme and the problems related to record loss and inconsistencies in healthcare settings whilst gratifying the standards for electricity economy, low latency and dependability. The purpose is to find out about and analyse the method of discovering imperative algorithmic protocols. The concern covers body charge analysis, scheme use and device overall performance monitoring. Additionally, the evaluation covers the WBAN system's per-sensor consumption (CUU) central manipulate unit time. The modelling was once carried out for a wi-fi channel physique radio channel, and an omnidirectional antenna was used for the newly delivered 402-405 MHz Medical Implant Communication Service (MICS). The scheme has four sensor nodes (five sensors related to a single collector), a single manipulate unit and a faraway PC for sign gathering and display. The entered facts are outfitted to the route loss model's post processing dimension statistics' result parameters. The effects and evaluation of the three conditions used to discover crucial processes are provided, and the findings are analysed in element as aspects of numerous imperative criteria. The learning from the consequences demonstrates that providing identities to all counselled structures resolves the route loss problem in WBAN and decreases the direction loss ratio between sender and recipient. The IKA scheme outperforms preceding strategies in phrases of route loss, and the consequences of using the receiving station in the backyard of the emergency room barring any route loss reveal IKA's efficacy. In conclusion, our findings result in a workable reply for the prohibition of MICS utilisation in a scientific setting.

\section{PATH LOSS IN WBAN}

With the use of simulation, route loss in WBAN and its effect on conversation are revealed [6]. The authors addressed the problem of statistic loss or variant in scientific implants and conversation waves in [7,8], [9], the place they investigated and produced consequences on the route loss mannequin in the MICS channel. In [5], [8], the authors mentioned in vivo verbal exchange channel loss introduced sensor units for machine monitoring and described a server-based architecture. With the world populace growing, the demand for satisfactory healthcare device functions in WBAN, especially for the aged who are more prone to fitness problems, is increasing. Numerous researchers have presented offerings to the elderly; for example, prototype structures using Bluetooth as a wi-fi physique region community (WBAN) to display vicinity and fitness status, as properly as smartphones geared up with accelerometers as intelligent central node, have been used to architect healthcare systems. This structure allows household participants or healthcare practitioners to obtain the right of entry to the place and fitness circumstance of sufferers in actual time via the Internet. ZigBee is utilised for low-data-rate purposes due to the fact of its decreased energy consumption than Bluetooth [10], [11]. To date, the foremost difficulty with WBAN is record loss or transmission corruption; new protocols or schemes are required to show the effectivity of receiving whole records without loss; the loss is brought on through free space impairment of the propagating sign (e.g. refraction), fading, absorption and reflection, amongst others. Additionally, it is based on the distance between the transmitter and reception antennas, the antenna's top and position, the propagation medium, such as moist or dry air, and the rural or city place in which the antenna is located, amongst different factors. 


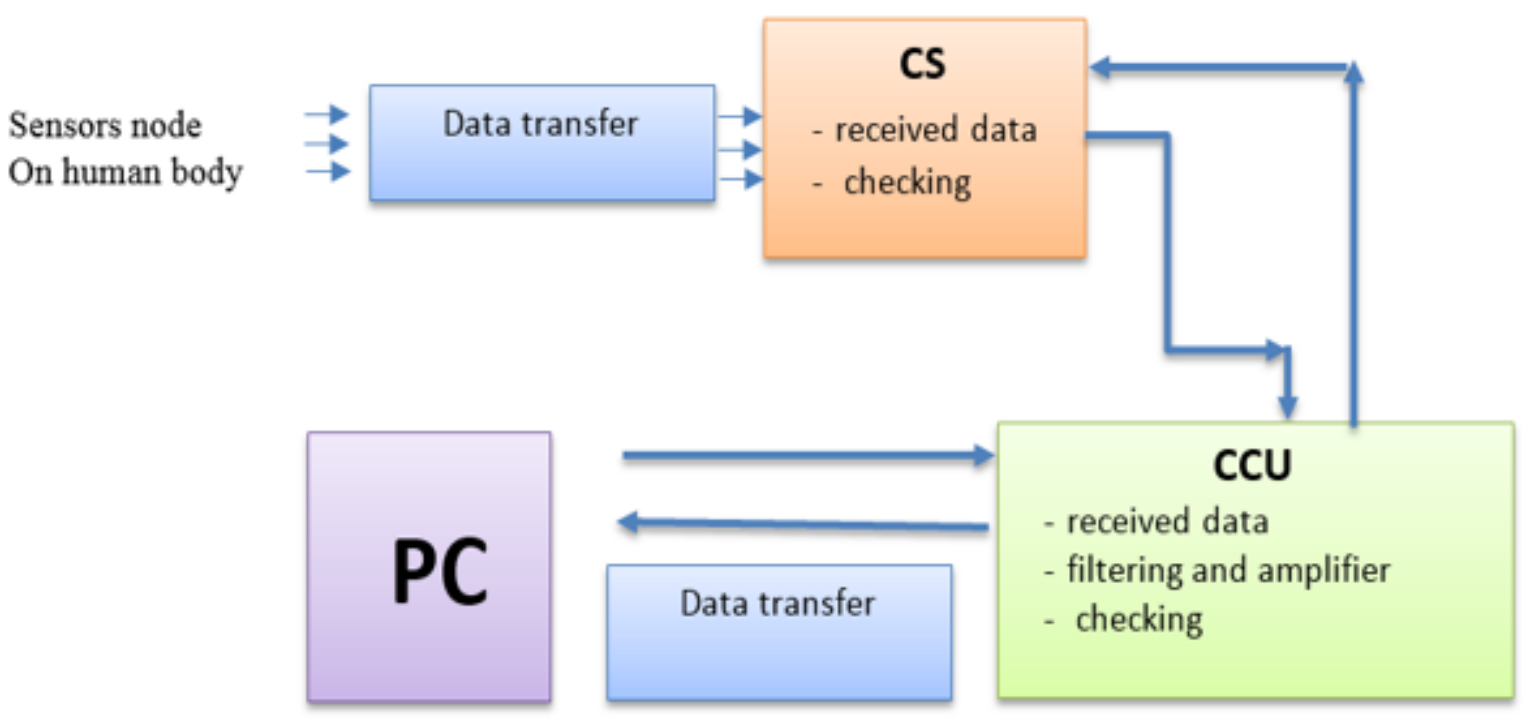

FIGURE 1. Three places for checking in the proposed model

\section{GENERAL STRUCTURE OF IDENTIFICATION KEY SCHEME}

When facts gathered via sensors and gear are transferred to far locations via wi-fi media, course loss is probable to occur. The figure below illustrates our proposed model's grasp station.

Path loss occurs for the duration of machine fitness monitoring owing to a multitude of variables, such as humidity, running frequency and bodily environment, such as distance between transmitter and receiver. Determining the key scheme's strategies and approaches is recommended. Determining the key protocol demonstrates the effectiveness of statistic transmission and reception in a clinical setting. To tackle the problem of information mismatch between what the receiving system receives and what the patient's sensor sends, the answer can cover the surroundings of three exceptional receiving stations. If one of them experiences direction loss, it can notify the monitoring birthday party receiving station, such as a medical doctor or nurse.

\subsection{AUTOMATISM OF MICS AND CCU IN SCHEME}

Previously used wi-fi statistic acquisition requirements such as Zigbee or Bluetooth are no longer compliant with scientific requirements due to their size, strength consumption and excessive interference from different units [3], [12], and such structures grew to be cumbersome and required affected person transport due to the thousands of sensors linked to the patient's body. As a result, this work uses the MICS band, an unlicensed, ultralow-power cell radio service, to transmit records in the guidance of diagnostic or therapeutic things to do related to implanted clinical gadgets [4]. Individuals and clinical practitioners can use ultralow to high-power scientific implanted gadgets such as pacemakers and defibrillators except interfering with different customers of the electromagnetic radio spectrum [9], [13] Our proposed format necessitates the use of several central controlled units (CCU) to organise the gathering of clinical statistics from the patient's body, which has the introduced gain of permitting the affected person to tour freely inside the clinic and use a single CCU for all sensors. The CCU is relocated to a more convenient area inside the emergency department, between 1 and 10 meters away. The CCU collects facts from the sensors, filters them to remove undesirable indicators and noise, amplifies them all through transmission, and finally, conducts assessments for direction loss based totally on the CCU table. The CCU is then linked via the MICS band to a neighbourhood PC, the place it indicates real-time information from the sensor nodes and shops it in a neighbourhood database in an identical manner as a faraway PC at a scientific middle would. The recorded records can be transmitted to the clinical centre's database on a habitual groundwork via the Internet. In hospitals with countless sufferers housed in the equal room, the applicable software programme applications need to be established in the CCU and on the neighbourhood PC to obtain physiological inputs from every patient's sensor. Record 
gathering takes place at predetermined time intervals distributed to the patients. The figure below shows the function of the CCU in our design.

\subsection{SCENARIO ENVIRONMENT OF SCHEME}

Before setting up the situation surroundings for the IKA scheme, the requirements for the WBAN situation for the number of research to illustrate the findings' efficacy are addressed in depth, as we learn the indispensable aspects of an environment-friendly scenario, such as antenna sorts and sensor types. Additionally, in the $2 \mathrm{~d}$ case, the surroundings are altered to permit the extension of our counselled answer through the new method. Our paper is executed utilising three eventualities involving sufferers in the emergency branch and the vicinity outside the emergency room. The eventualities assume that all situations appear in the identical clinic and that all sufferers are in an equal location. However, the receiving units are now not all positioned in the identical region in all three situations. These three eventualities are tackled in depth. As stated previously, WBAN problems occur when information is transferred between sending and receiving locations and facts are misplaced or altered, which is the main difficulty owing to the type of protocol used with WBAN or the harsh bodily environment, or information is misinterpreted through the receiver. The new scheme's core premise is to supply unique statistics about the sensor, such as the sensor's kind, functioning and conversion rate. Future developments in microchips and digital devices will generate new thoughts for the vicinity and resource researchers in growing future WBAN routing protocols and schemes. For this reason, using the reminiscence of the chip sensor, which is reachable due to the circuit's architecture is preferred here, and the sort of reminiscence is successful in storing and modifying data. This paper anticipates that a sensor has a special reminiscence and its reason is to acquire records from different sensors. This sensor is referred to as the collector sensor (CS), and in this phase, the new protocol's first step is provoked. Each sensor transmits statistics to the CS, and the sensor desk is stored in the CS memory. In this manner, whether any information is misplaced or if the facts are transferred between the sensor node and the CS change can be effortlessly decided. The $2 \mathrm{~d}$ stage is to switch the information to the CCU, which then transmits it to the neighbourhood private PC and the clinical centre's receiving station. Our strategy is suitable for special conditions encountered in the emergency room (one affected person to one receiver, more than one sufferer to one receiver and a couple of sufferers to a couple of receivers). The figure below depicts a situation, in which our novel routing protocol answer is implemented.

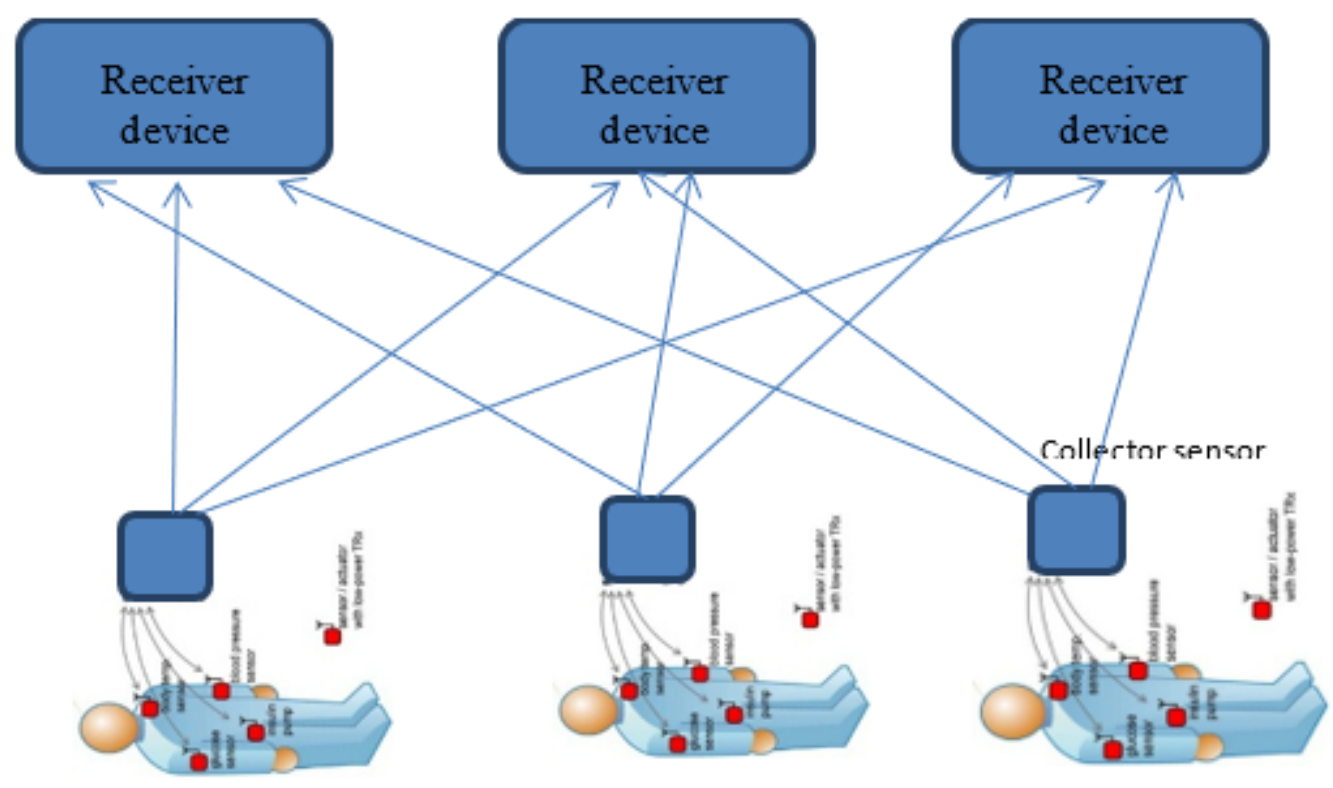

FIGURE 2. Main diagram of three cases

Precise situations in which to practice and check the new schemes for acquiring information from affected person sensors are selected due to the incapability of any technique to be used in large location surroundings and to ensure suitable results. Additionally, the simulations aid us in comprehending the enhancements to statistic transmission and reception, and in demonstrating the efficacy of the protocols employed in WBAN. 


\subsection{SPECIFICATION OF SENSOR IN OUR PROPOSEID SYSTEM}

For smart monitoring, the sensing factors in the proposed gadget are light weight, compact size and minimal strength [14]. These sensors reveal human integral signs, bodily herbal processes and herbal approaches in actual time. A rising want for misplaced statistics in that location is observed, which will require much less energy and result in a longer battery life. The counselled strategy satisfies this standard entirely. It is viable to accomplish excessive statistic price transmission through making the physique sensors like-minded with the underlying science [15]. The sensor's foremost characteristic is to acquire indispensable indicators from the human physique and transmit them to the CCU; in general, all sensor nodes contact with the equal CCU, and those facts are prefixed with an identifier that serves to perceive the supply of the data, which is the human body's unique sign [16]. Typically, the indicators emanating from the human physique are susceptible or nonexistent,FirstlyremoveNextanalogue-to-digital conversion step seriously changesThe microprocessor due to this fact strategies and shops the digital signal. After obtaining thesthemthrougha transmitter [2], [17] The node for measuring the pulse charge is provided. The microcontroller PIC16F877 and the transceiver AMIS-5210 are selected for this venture owing to their low manage utilisation, compact size, appropriateness for use in the MICS band and capability to organize physiological data. The temperature and coronary heart fee sensor hubs are built with the use of well- known printed circuit boards. As a result, the units are interchangeable. The CCU desires in our advised device are for minute controllers and far-off handset chips to prepare all workouts, such as sensor nodes allotted throughout the human body. According to [18], the identical transceiver chip from AMI semiconductor (AMI52100 IC) for the CCU and sensor nodes is used, as nicely as the microcontroller PIC16F87, to reveal that they are well suited in a single machine and to gain the intention of our paper, which is to obtain a result in which the distance between the CCU and the sensor can be changed without difficulty. This vicinity can be somewhere in the emergency room, and the wi-fi distance should be between 1 and 10 meters when using the MICS band. The important points of how to use the MICS band are addressed in depth later in Section 3.5 of this chapter, and the connection of the CCU is discussed in Section 3.5.1. Our investigation no longer uses random gadgets because the coordination of the system's components is indispensable in acquiring a correct look at findings. The following sketch illustrates the relationships between our model's most important components:

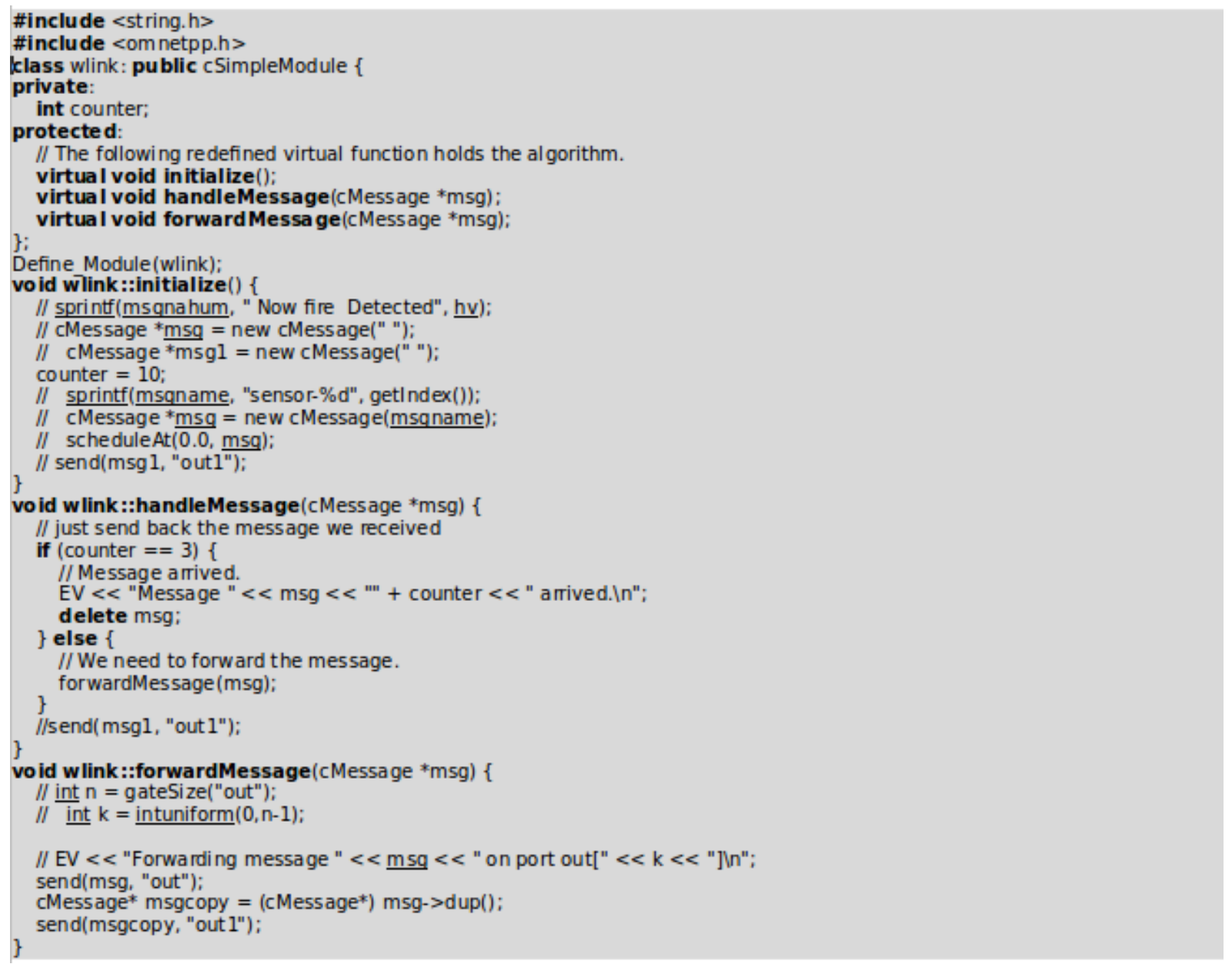


The throughput metering is the delay, data rate and path loss factor, programmed as below:

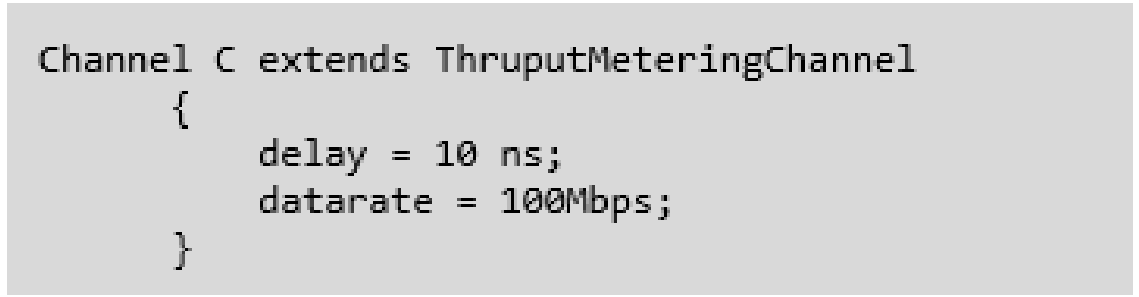

Our device includes a range of gadgets, which includes minute sensors implanted in or around the physique to display the patient. The information is transmitted to the series sensor at the beginning, validated for any lacking simple guidelines and then transferred to the CCU gadget over the MICS band. The CCU reminiscence is programmed with a desk referred to as the CCU desk that performs assessments for lacking facts. In case of lacking data, the CCU software programme reinterrogates the facts from the series sensor. The receiving station (i.e. the far- away PC) can show all the obtained facts on a person's show photograph (GUI) and keep all the information in the clinical centre's database system. The human physique is modelled in our programming as follows:

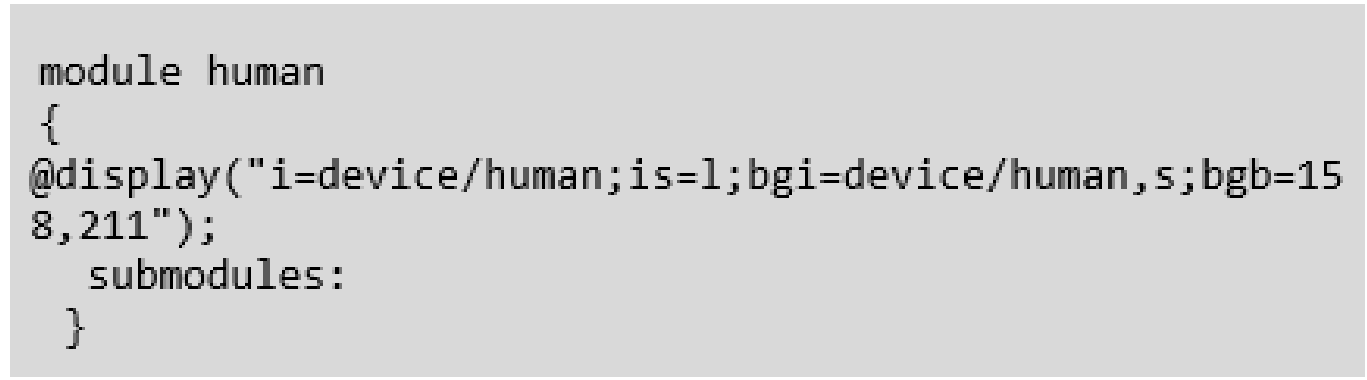

\section{PROCESS OF DECREASE PATH LOSS IN IKA}

Path loss is the distinction between dispatcher and obtained strength (in decibels), which displays sign attenuation owing to free house propagation, reflection, bypassing and scattering. The price range for the connection is calculated. After calculating the hyperlink budget, the facts of the first CS and sensor can be entered into the CCU, and the distance between the sensor and the CS is obtained. It is coded in the following manner.

The hyperlink budgets S1, S2, S3, S4 and S5 are calculated, and then the effects are documented in the role sensor field:

Table 1. Distance for each sensor with CS in proposed scheme calculation link

\begin{tabular}{|c|}
\hline Position of sensor $/$ link budget to CS \\
\hline Head $/ 5 \mathrm{~cm}$ \\
\hline Heart $/ 4 \mathrm{~cm}$ \\
\hline Arm $/ 3.5 \mathrm{~cm}$ \\
\hline Neck $/ 4 \mathrm{~cm}$ \\
\hline armpit $/ 3 \mathrm{~cm}$ \\
\hline
\end{tabular}

The programming of CS and CCU table is shown below: 


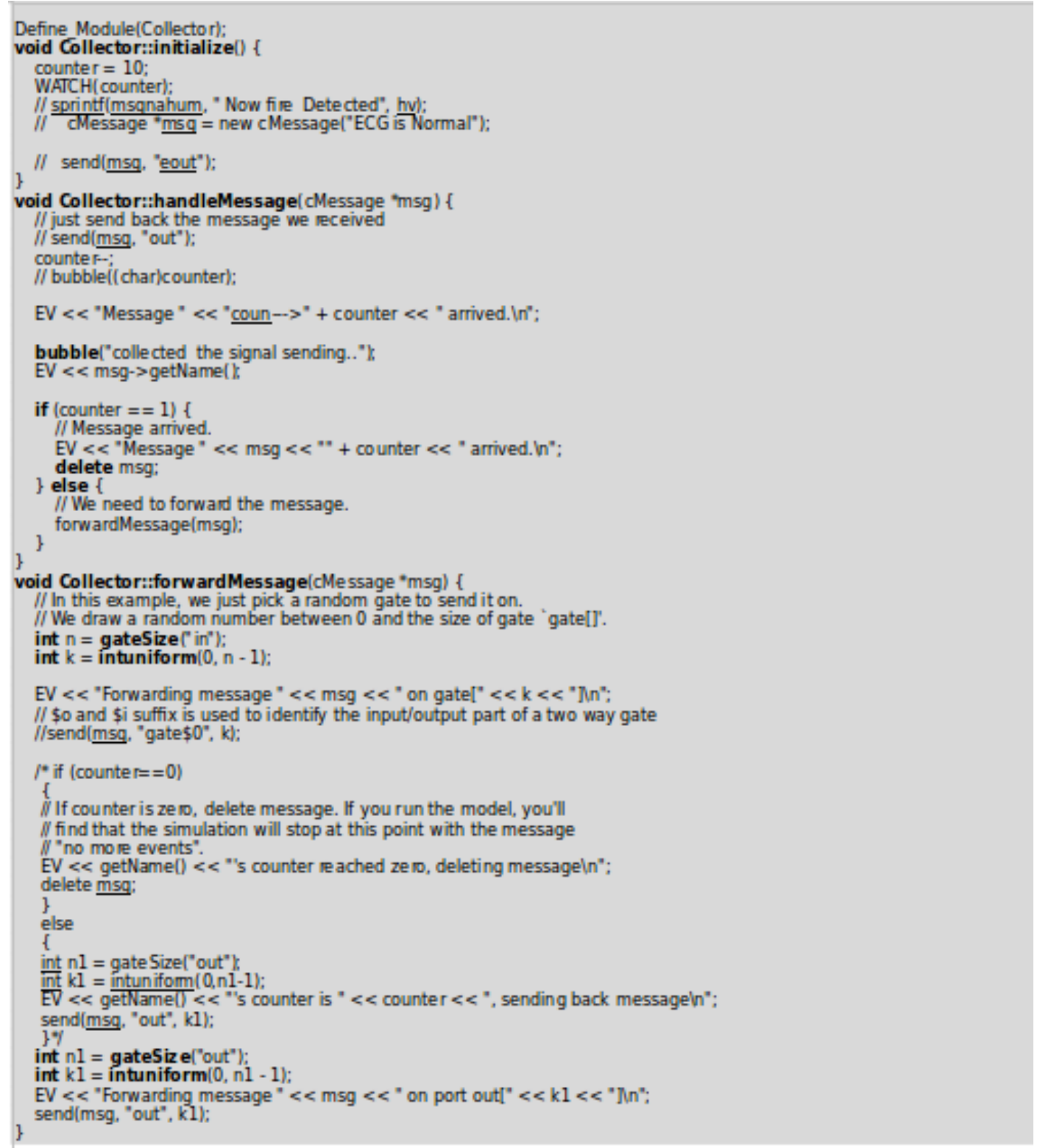

For the CCU, the important configuration of the thought on the CS is established on it. It receives facts from the CS, and the statistics need to be checked in the CS before sending. In case of a course loss, the CCU tests the information again. As stated previously, programming is based on the latency of the measured direction loss and the rate factor. The sensor's identification simplifies this procedure, as it will be utilised for CS, CCU and PC.

\section{DESCRIPTION OF DATA ON COLLECTOR SENSOR}

The central notion has a programmable reminiscence accessed by using a one-of-a-kind sensor known as the collector sensor (CS). The CS performs the equal feature as the WSN's receiver and gathers statistics from sensors close by. Following collection, the desk is created as a CS software examination for route loss, and then inquiries about records loss are made from the sensor. The utilisation distribution of the sensors that are hooked up on the series sensors is proven in Table 2. The following desk lists the sensors on which series sensors have to be placed:

The position of the CS in the human body in our design is shown below:

Figures 4, 5 and 6 show the first, second and third scenarios, respectively. 
Table 2. Distribution to collector sensor

\begin{tabular}{|c|c|c|c|c|}
\hline $\begin{array}{c}\text { Sensor } \\
\text { no }\end{array}$ & Sensor job & $\begin{array}{c}\text { Position of sensor } \\
/ \text { link budget }\end{array}$ & The vibration & $\begin{array}{c}\text { Received } \\
\text { data }\end{array}$ \\
\hline Sensor 1 & Brain signal & Head $/ 5 \mathrm{~cm}$ & Every $2 \mathrm{Sec}$ & Yes $/$ no \\
\hline Sensor 2 & Heart signal & Heart $/ 4 \mathrm{~cm}$ & Every $5 \mathrm{Sec}$ & Yes $/$ no \\
\hline Sensor 3 & Pressure & Arm $/ 3.5 \mathrm{~cm}$ & Every $15 \mathrm{mins}$ & Yes $/$ no \\
\hline Sensor 4 & Blood flow & Neck $/ 4 \mathrm{~cm}$ & Every $10 \mathrm{mins}$ & Yes $/$ no \\
\hline Sensor 5 & temperature & armpit $/ 3 \mathrm{~cm}$ & Every $15 \mathrm{~min}$ & Yes $/$ no \\
\hline
\end{tabular}

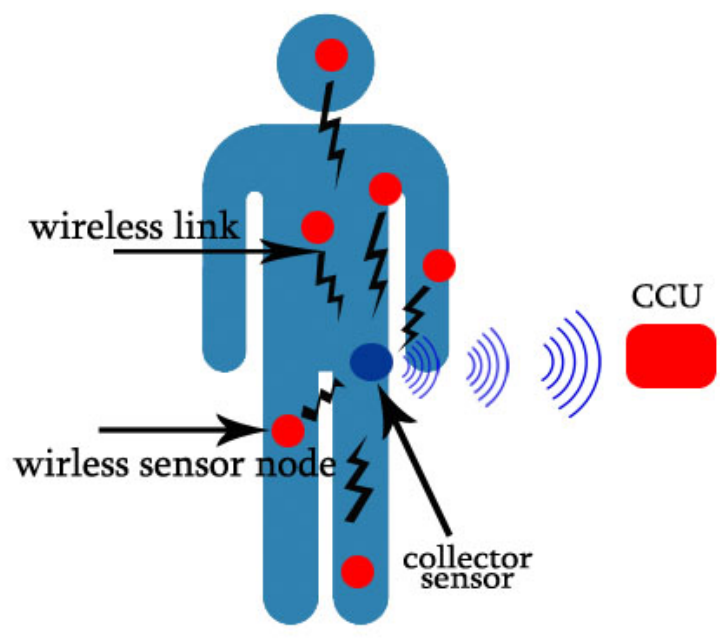

FIGURE 3. Sensors' collector sensor locates in the proposed scheme with

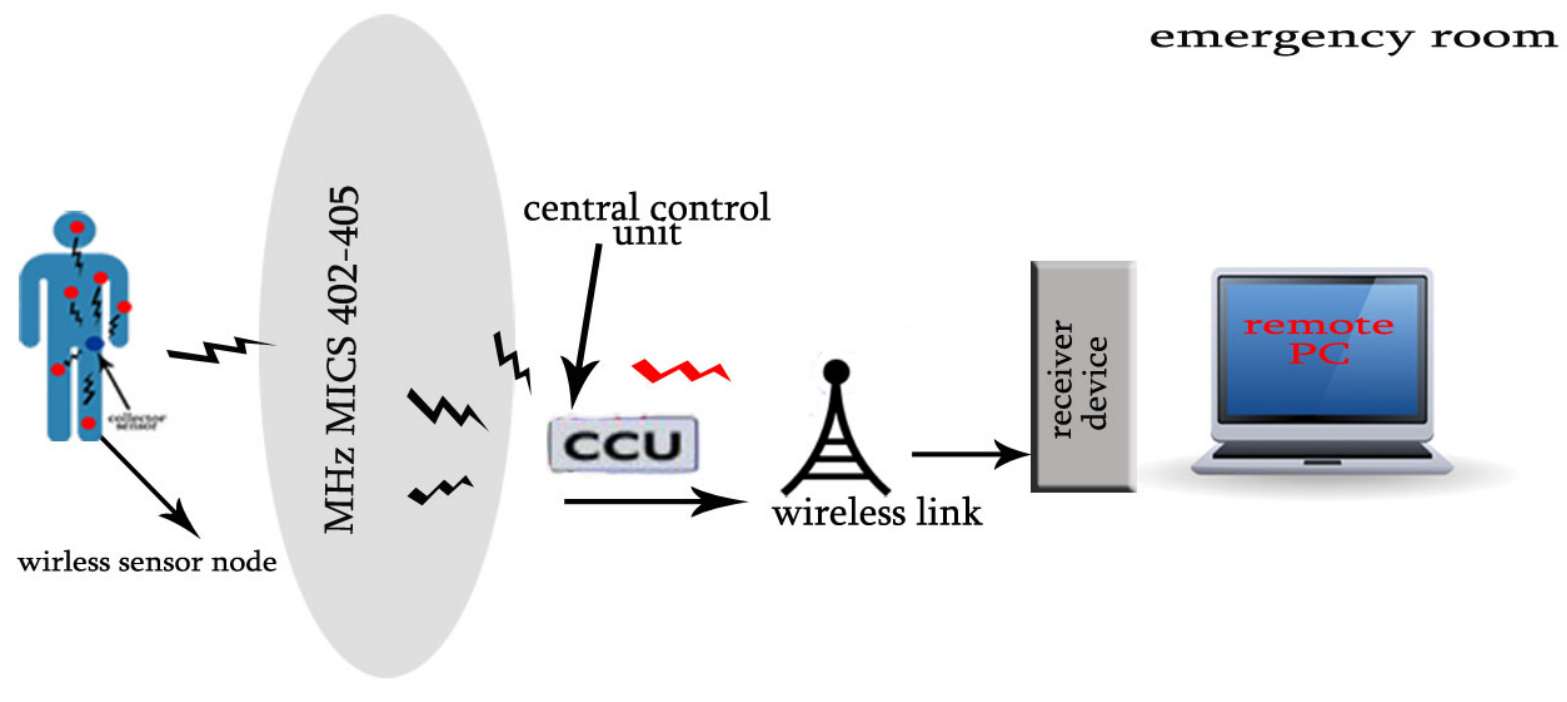

FIGURE 4. Diagram of first scenario: three cases send to one receiver station 


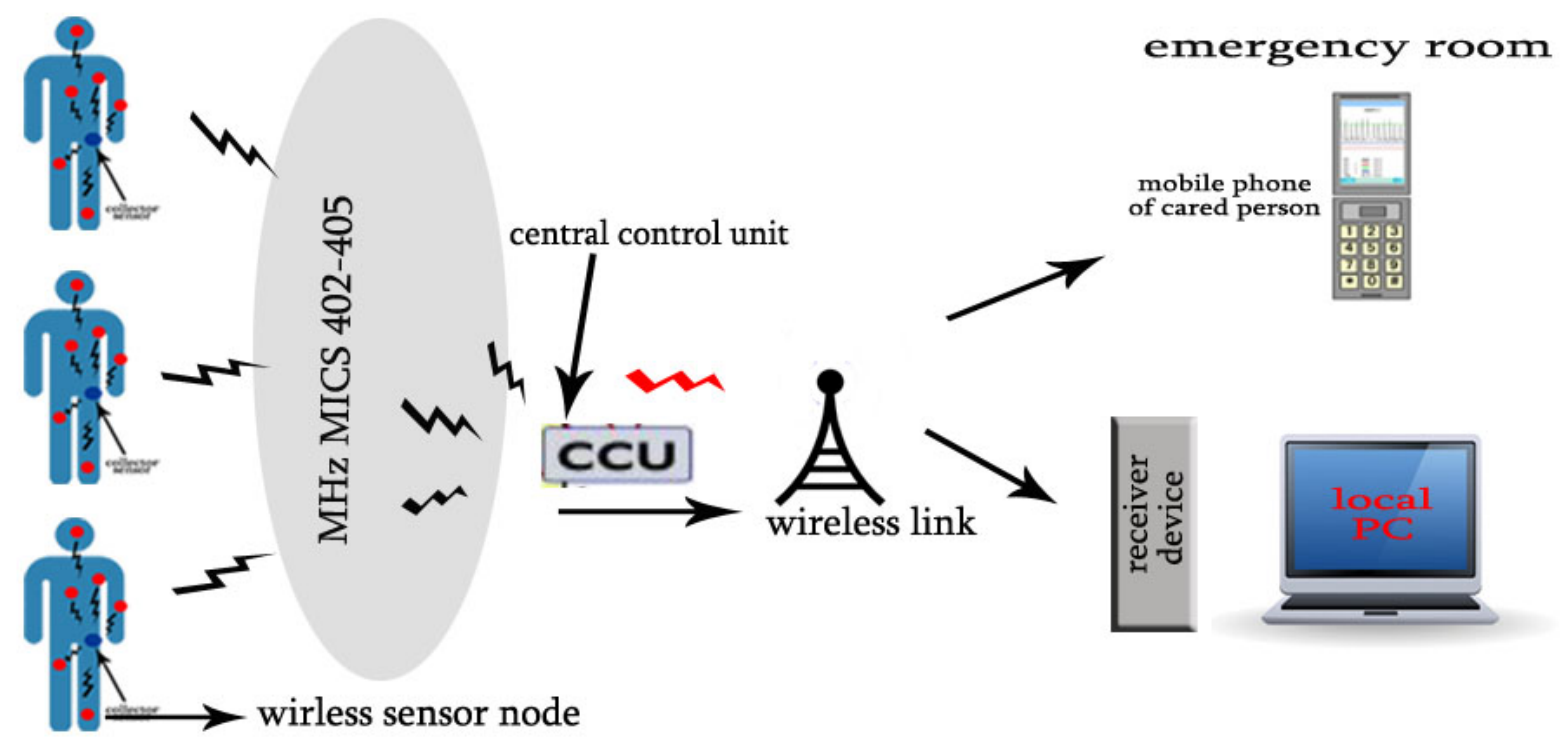

FIGURE 5. Diagram of second scenario: the cases send to two receiver stations

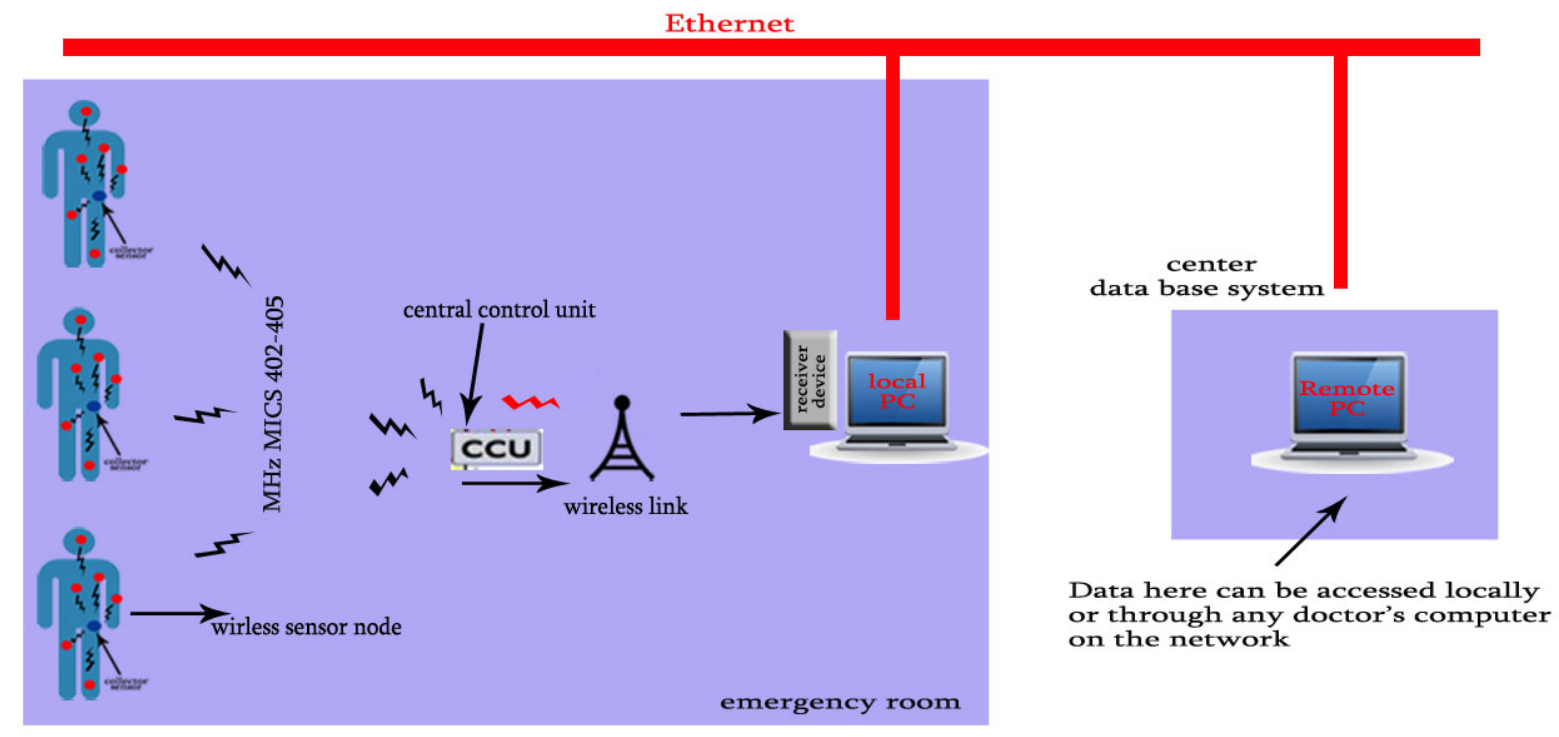

FIGURE 6. Diagram of third scenario: the cases send to three receiver stations 


\section{PERFORMANCE ANALYSIS OF SCHEMES}

This part analyzes the findings for three conditions earlier than to and following the use of IKA. Before enforcing our cautioned method, it is integral to estimate the route loss thing the use of the RSS localization technique, as prior lookup used this direction loss issue to quantify course loss. The findings for the first, second, and third conditions earlier than and after using IKA are proven in results, relying on traits such as course loss, latency, and facts rate. The discount in route loss ratio following IKA implementation is confirmed in three scenarios, with point-by-point inspection and region readings, and the first scenario's environmental simulation and emergency room wishes are certain in Chapter four the following desk summarizes the findings of the $2 \mathrm{~d}$ situation making use of route loss, delay, and charge statistics metrics each earlier than and after IKA was once used. Additionally, when our advised answer IKA is used, we see a limit in the course loss ratio, demonstrating the effectiveness of our strategy in lowering route loss between transmitting and receiving stations in the scientific surroundings domain.

\section{THE CONCLUSION}

The literature gaps highlighted in this learn about are that modern-day options for course loss in Wireless Body Area Network (WBAN) do no longer fulfill all fitness monitoring gadget wants and nonetheless have route loss concerns and that our counseled answer is missing facts at the receiving station. According to the identities assigned to all MICS band-based sensors, their radio connections with implantable clinical units are increasing frontiers in our appreciation and prevention of human disease. The paper proposes options for three awesome situations involving the positioning of receiving stations. The simulation consequences in OMNET++ point out the diminished course ratio in WBAN, and the utilization of OMNET++ allows conversation in our design, which employs INT frames.

\section{ACKNOWLEDGMENT}

This paper extends the study [5] that explains the scheme used in transfer patient data and the paper mentioned for the same author of this paper. This paper also depends on studies [1], [3], [15], [19] that the same author published before.

\section{CONFLICTS OF INTEREST}

The authors declare no conflict of interest.

\section{REFERENCES}

[1] I. Al Barazanchi, H. R. Abdulshaheed, M. Safiah, and B. Sidek. A Survey : Issues and challenges of communication technologies in WBAN. Sustain Eng Innov. 2020; vol. 1, no. (2): pp. 84-97.

[2] I. Al Barazanchi, H. R. Abdulshaheed, M. Safiah, and B. Sidek. Innovative technologies of wireless sensor network : The applications of WBAN system and environment. Sustain Eng Innov. 2020; vol. 1, no. (2): pp. 98-105.

[3] A. Shibghatullah and I. Al Barazanchi. A survey on Central Control Unit ( CCU ) in WBAN. Int Symp Res Innov Sustain. 2014; vol. 14: pp. $15-16$.

[4] D. Zou and S. Ma. Satellite Navigation and Communication Integration Based on Correlation Domain Indefinite Pulse Position Modulation Signal. Wirel Commun Mob Comput;2021: pp. 2021-2021.

[5] I. Al Barazanchi, H. R. Abdulshaheed, S. A. Shawkat, and S. R. Binti. Identification key scheme to enhance network performance in wireless body area network. Period Eng Nat Sci. 2019; vol. 7, no. (2): pp. 895-906.

[6] H. R. Abdulshaheed, S. A. Binti, and I. I. Sadiq. Proposed a Smart Solutions Based-on Cloud Computing and Wireless Sensing. Int J Pure Appl Math. 2018; vol. 119, no. (18): pp. 427-449.

[7] S. B. Tambe and S. S. Gajre. Cluster-based real-time analysis of mobile healthcare application for prediction of physiological data. J Ambient Intell Humaniz Comput. 2018; vol. 9, no. (2): pp. 429-445.

[8] R. Saha, S. Biswas, S. Sarma, S. Karmakar, and P. Das. Springer US.

[9] T. Arefin, M. H. Ali, and A. K. M. F. Haque. Scenario Based Transmission Power Control (TPC) Analysis over Wireless Body Area Network (WBAN). Commun Netw. 2021; vol. 13, no. (03): pp. 93-110.

[10] I. Abdulshaheed, H. R., Yaseen, Z. T., Salman, A. M., \& Al_Barazanchi. An Evaluation study of WiMAX and WiFi on Vehicular Ad-Hoc Networks ( VANETs ). IOP Conf Ser Mater Sci Eng Pap. 2020; vol. 3, no. (12): pp. 1-7.

[11] H. H. A. and H. R. A. I. Al-Barazanchi, Z. A. Jaaz. Practical application of IOT and its implications on the existing software. Conf Electr Eng Comput Sci Informatics (EECSI). 2020; vol. 2020: pp. 10-14.

[12] S. S. Oleiwi, G. N. Mohammed, and I. Al-barazanchi. Mitigation of packet loss with end-to-end delay in wireless body area network applications. Int J Electr Comput Eng. 2022; vol. 12, no. (1): pp. 460-470.

[13] I. Al Barazanchi, W. Hashim, A. A. Alkahtani, and H. R. Abdulshaheed. Survey : The impact of the Corona pandemic on people, health care systems, economic : Positive and negative outcomes. The Role of Intellectual in Achieving Sustainable Development after the COVID-19 and the Economic Crisis Conference RICSDCO19EC. 2021; pp. 125-125. 
[14] R. K. Naji, M. G. Younis, and Mohammad Naeemullah. The Dynamics of a Single Species in a Polluted Environment. ” Iraqi J Comput Sci Math. 2020; pp. 1-12.

[15] I. Al Barazanchi, A. S. Shibghatullah, and S. R. Selamat. A New Routing Protocols for Reducing Path Loss in Wireless Body Area Network ( WBAN ). J Telecommun Electron Comput Eng Model. 2017; vol. 9, no. (1): pp. 1-5.

[16] A. M. Salman. Implementation of Mobile robotics in Autonomous Mobility tracking Robot, 2020; vol. 29 , no. 4, pp. 448457.

[17] M. S. Akbar, H. Yu, and S. Cang. Delay, Reliability, and Throughput Based QoS Profile : A MAC Layer Performance Optimization Mechanism for Biomedical Applications in Wireless Body Area Sensor Networks. J Sensors. 2016; vol. 2016, no. (7): pp. 17-17.

[18] N. Sabah, A. Sagheer, and O. Dawood. Survey: (Blockchain-Based Solution for COVID-19 and Smart Contract Healthcare Certification). ” Iraqi J Comput Sci Math. 2021; pp. 1-8.

[19] I. Al Barazanchi. An Analysis of the Requirements for Efficient Protocols in WBAN. J Telecommun Electron Comput Eng. 2014; vol. 6: pp. 43-43. 